Family Medicine and Community Health

\section{Telemedicine, the current COVID-19 pandemic and the future: a narrative review and perspectives moving forward in the USA}

To cite: Kichloo A, Albosta M, Dettloff $\mathrm{K}$, et al. Telemedicine, the current COVID-19 pandemic and the future: a narrative review and perspectives moving forward in the USA. Fam Med Com Health 2020;8:e000530. doi:10.1136/fmch-2020-000530

Check for updates

(C) Author(s) (or their employer(s)) 2020. Re-use permitted under CC BY-NC. No commercial re-use. See rights and permissions. Published by BMJ.

${ }^{1}$ Internal Medicine, Central Michigan University College of Medicine East Campus, Saginaw, Michigan, USA

${ }^{2}$ Family Medicine, Samaritan Medical Center, Watertown, New York, USA

${ }^{3}$ Medicine, Geisinger Commonwealth School of Medicine, Scranton,

Pennsylvania, USA

${ }^{4}$ Internal Medicine, Hurley Medical Center, Flint, Michigan, USA

${ }^{5}$ Nephrology, Westchester Medical Center, Valhalla, New York, USA

Correspondence to Dr Michael Albosta; albos1ms@cmich.edu

\section{ABSTRACT}

A narrative review was conducted to examine the current state of the utilisation of telemedicine amid the current COVID-19 pandemic and to evaluate the benefits of continuing telemedicine usage in the future. A literature review was performed for articles related to telemedicine. Databases including PubMed, Google Scholar, Cochrane Library and Ovid MEDLINE were searched. Three reviewers independently performed article selection based on relevance to our topic. We included all articles between 1990 and 2020 related to telemedicine using the following keywords: 'telemedicine', 'telehealth', 'policy', 'COVID-19', 'regulation', 'rural', 'physical examination', 'future'. A total of 60 articles were identified, and through careful selection we narrowed the final number of articles to 42 based on relevance to our topic. Telemedicine has been rapidly evolving over the past several decades. Issues with regulation and reimbursement have prevented its full immersion into the healthcare system. During the current pandemic, Centers for Medicare and Medicaid services have expanded access to telemedicine services. The advantages of telemedicine moving forward include its cost-effectiveness, ability to extend access to specialty services and its potential to help mitigate the looming physician shortage. Disadvantages include lack of available technological resources in certain parts of the country, issues with security of patient data, and challenges in performing the traditional patient examination. It is critically important that changes are made to fully immerse telemedicine services into the healthcare landscape in order to be prepared for future pandemics as well as to reap the benefits of this service in the future

\section{INTRODUCTION}

Telemedicine is a service that is rapidly evolving to provide increased access to high-quality healthcare that is efficient and cost-effective, especially in the midst of the current COVID-19 pandemic. According to the Centers for Medicare and Medicaid Services (CMS), ${ }^{1}$ telemedicine is 'a service that seeks to improve a patient's health by permitting two-way, real-time interactive communication between the patient and the physician at a distant site'. Although similar, the terms 'telehealth' and 'telemedicine' should not be used interchangeably. Telehealth refers to 'the use of telecommunications and information technology (IT) to provide access to health assessment, diagnosis, intervention, consultation, supervision and information across distance'. ${ }^{1}$ Therefore, telehealth can be considered a more broad concept of telemedicine that includes technology used to collect and transmit patient data such as telephones, email and remote patient monitoring (RPM) devices for the purposes of providing health education or ancillary healthcare services. ${ }^{12}$ Over the past several decades, improvements in technology have dramatically increased the accessibility and quality of care that is available digitally. Despite this, telemedicine has yet to be widely implemented due to heavy regulatory laws and a lack of supportive payment structures. ${ }^{3}$ In the face of the current pandemic, providers have been forced to increase their utilisation of telehealth services at the expense of traditional face-to-face patient encounters. Over the past several years, researchers have been exploring the advantages and disadvantages of telemedicine compared with traditional patient encounters. During the current pandemic, telemedicine has the potential to greatly improve access to quality, affordable care for patients while maintaining physical distancing for the safety of both patients and providers. In addition to virtual visits, text, email and mobile phone applications as well as data from wearable devices can be used to share information between patients and clinicians. ${ }^{3}$ In this article, we aim to discuss the evolution of the telemedicine landscape, 
its utilisation during the current pandemic and how we expect this technology to be implemented in the postpandemic world.

\section{Background of telemedicine}

The origins of telemedicine can be traced as far back as the use of ancient hieroglyphs and scrolls to share information about health related events such as outbreaks or epidemics. ${ }^{4}$ Furthermore, some societies were known to use smoke signals to warn nearby cities of sickness. ${ }^{4}$ As we entered into the 19th century, the advent of the telephone and the typewriter transformed the way that patients and physicians shared health information. ${ }^{45}$ During the Civil war, the telegraph was used in order to transmit information regarding casualties and to call for medical supplies. ${ }^{5}$ The telephone was used during the Korean and Vietnam wars in order to dispatch medical teams. ${ }^{5}$ The 1950 s brought the development of the television, and in 1959 the Nebraska Psychiatric Institute began using videoconferencing for telepsychiatry.

The National Aeronautics and Space Administration (NASA) played a major role in the development of telemedicine as we know it today. The need for medical care during space travel allowed physicians to monitor the vital signs of astronauts during flights, as well as provide diagnostics and treatment in-flight. ${ }^{5}$ Additionally, NASA helped provide telemedicine services to various rural locations in states such as Minnesota, New Hampshire, Maine, Alaska, Arizona and Washington during the 1970s and 1980s. ${ }^{4}$ This was a part of several federally funded projects to determine the current capabilities of telemedicine equipment and the clinical applicability of telemedicine as a service. ${ }^{4}$ In the 1990 s, the internet changed the way we used telemedicine services. The internet has allowed for great improvements in sharing of medical images such as X-rays or scans, vital signs, ECG and realtime audio and video interaction.

Today, with the advancement of mobile and electronic technologies, telemedicine is more accessible than ever before. According to a 2019 report by the Pew Research Center ${ }^{6} 90 \%$ of Americans use the internet. Furthermore, $81 \%$ of Americans own a smartphone, nearly $75 \%$ own desktop or laptop computers and roughly $50 \%$ own tablet computers or e-readers. ${ }^{7}$ This increase in access to mobile technologies has been critical for the advancement of telemedicine. Because of this, the use of the internet is now mainstream in healthcare. The use of electronic medical records allows for the storage and access of medical information for both patients and providers. Through these services patients can view results, refill medications and send messages directly to their physician. In addition, we now have the ability to interact face to face with providers in real-time via live video, also known as synchronous telemedicine. We also have the ability to share imaging, labs or examination results so that these can be interpreted at a later date, referred to as Store-and-Forward or asynchronous telemedicine. Lastly, we use telemedicine measurement devices such as smartphone cameras, digital stethoscopes, ophthalmoscopes, otoscopes and wearable biosensors to further improve the telemedicine experience for both patients and providers. The use of these mobile devices to record and transmit data to healthcare professionals is referred to as RPM. ${ }^{2}$

\section{Telemedicine before COVID-19}

The utilisation of telemedicine has been rapidly increasing in the USA. From 2010 to 2017, the percentage of US hospitals that connect with patients through the use of video and other technology has increased from $35 \%$ to $76 \% .^{8}$ The American Medical Association ${ }^{9}$ further reported that telemedicine insurance claims increased by $53 \%$ from 2016 to 2017 . This is likely due to the increasing efficacy of telemedicine, as today physicians are able to deliver more and more of their services virtually.

The utilisation of telemedicine can be stratified in several ways, such as by specific services or specialty care. For example, a specific telemedicine service, Telestroke, has become one of the largest care providers for patients with stroke in the USA since its introduction in $1999 .{ }^{10}$ When considering the use of telemedicine by different specialties, it has been documented that certain medical specialties use telemedicine more than others. Researchers have found that radiologists, psychiatrists and cardiologists use telemedicine the most, at rates of $39.5 \%, 27.8 \%$ and $24.1 \%$, respectively. ${ }^{11}$ The specialists who use telemedicine the least are allergists/immunologists, gastroenterologists and obstetrician/gynaecologists at rates of $6.1 \%, 7.9 \%$ and $9.3 \% .^{11}$

Despite the rise in utilisation of telemedicine services, legal and regulatory challenges prevent its further expansion. The Office of the United Nations High Commissioner for Human Rights ${ }^{12}$ lists six key aspects of the right to health: accessibility, availability, participation, accountability, acceptability and good quality. Medicaid has deemed telemedicine to be an acceptable alternative to the more traditional face-to-face patient encounter, but further laws and regulations governing the other five key aspects are controlled individually by the state. ${ }^{1}$

Currently, all 50 states' and Washington DC's laws, policies and regulations on telemedicine differ significantly. Specific regulatory issues in the areas of coverage, payment, licensure, credentialing, online prescribing, medical malpractice, privacy and security, and fraud and abuse will determine how providers can offer specific telehealth services. ${ }^{8}$ Although many states use similar language in their policies, there are noticeable differences which create a confusing environment for telemedicine participants. ${ }^{13}$

While there are differences in policy and ambiguous verbiage between states, likely because each state defines its Medicaid policy parameters, several trends are seen. For example, as of February 2020, Medicaid fee-for-service provides reimbursement for some form of live video in all 50 states and Washington DC. ${ }^{13}$ However, only 16 state Medicaid programmes provide reimbursement for storeand-forward services, only 23 states reimburse for RPM 
services, and 10 states reimburse for all three with some limitations. ${ }^{13}$ Laws governing private payer telemedicine reimbursement policies are in effect in 42 states and Washington DC with a few states requiring that telemedicine reimbursement be equal to the reimbursement if the same service were to be delivered in person.

When compared with other countries including the European union, Korea and Japan, the USA is using telemedicine services at much higher rates. ${ }^{8}{ }^{14}$ As of 2012, $31 \%$ of hospitals and $15 \%$ of outpatient clinics were using telemedicine services in the European Union. ${ }^{14}$ In Japan, telemedicine is only used as an adjunct to traditional in-person visits for patients with chronic conditions or incurable diseases. ${ }^{14}$ This is because insurance companies do not accept claims for preventative or health consultation telemedicine visits unless quality of care is proven to be improved. ${ }^{14}$ As of $2013,12.5 \%$ of hospitals in Japan were using teleradiology services, $6.1 \%$ used telepathology and only $1.3 \%$ were using telemedicine for home patients. ${ }^{14}$ Lastly, in Korea, the implementation is only around $0.1 \%$ as of 2013-2014. ${ }^{14}$ According to Medical Law in Korea, telemedicine can be used for the purpose of improving access to care in remote areas, as well as for management of chronic disease and for patients who are disabled. ${ }^{14}$ In order to use these services, however, the patient must first see the physician in-person, and only then can telemedicine be used in conjunction with regular visits to monitor patient progress. ${ }^{14}$

Telemedicine is rapidly advancing and the demand for this service is increasing in the face of the current pandemic. As such, government and state regulations need to quickly adapt to the increased need for telemedicine and maintain its ability to provide the six key aspects to humans' right to health. This is a unique time for America and telemedicine policy needs to adapt more quickly than ever during the COVID-19 pandemic.

\section{METHODS}

A literature review was performed for articles related to telemedicine. We used PubMed, Google Scholar, Cochrane Library and Ovid MEDLINE to search published articles between the years 1990 and 2020 . Searches through the references of retrieved articles were also performed. In addition, we reviewed the web pages of various professional organisations including the American Telemedicine Association and the CMS for information and data relative to the topic of interest. We used the following keywords: 'telemedicine', 'telehealth', 'COVID19', 'policy', 'regulation', 'future', 'rural', 'physical examination'. A total of 60 articles were reviewed, and through careful selection we narrowed the final number of articles to 42. Three reviewers were responsible for performing article selection based on relevance to our topic. Inclusion criteria consisted of articles written between 1990 and 2020, published works that were available in English, and articles related to telemedicine in all settings. We excluded duplicates, abstracts, non-English articles, as well as those that were unpublished works or those not related to telemedicine. See figure 1 for details regarding
Query: Terms "telemedicine" OR "telehealth" OR "COVID-19" OR "policy" OR "regulation" OR "future" OR "rural" OR "physical exam" were searched using PubMed, Google Scholar, Cochrane Library, Ovid Medline.
Web pages of American Telemedicine Association and the Centers for Medicare and Medicaid services were reviewed for information and data related to telemedicine.

Three reviewers selected articles based on relevance to the topic. In addition, references of selected studies were reviewed for articles of relevance.

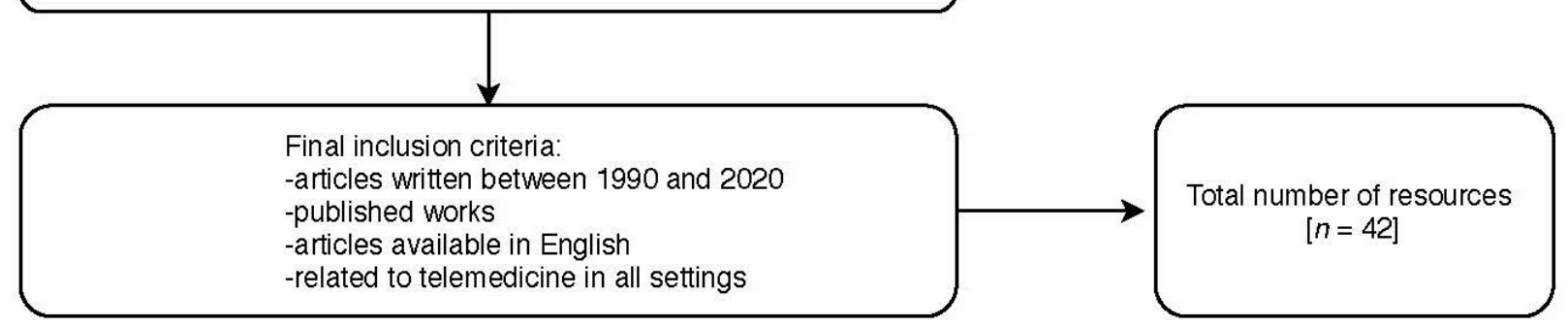

Figure 1 Article selection flowchart describing the process of literature review. 
query, study selection and inclusion/exclusion criteria used during literature review.

\section{DISCUSSION}

\section{Increased utilisation and need of the hour}

Telemedicine has become a means of 'forward triage,' which is when patients are triaged before they ever visit an emergency department, amid the COVID-19 pandemic. Particularly, direct-to-consumer, which is also referred to as on-demand telemedicine has become a means by which patients can be screened despite being self-quarantined. This means of triage maintains patient-oriented care while protecting patients and healthcare providers. For patients who may have COVID-19, telemedicine has been used to assess respiratory symptoms, which may be part of the early presentation of COVID-19 infection. ${ }^{15}$

The utilisation of telemedicine has had positive impacts in the public health emergency beyond facilitating triage, including allowing the rapid deployment of large numbers of healthcare providers and the providing of services when local hospitals and healthcare centres are unable to meet demand. Telemedicine has been a means of providing healthcare information not only to infected people but also to non-infected people during this infectious pandemic. ${ }^{16}$ Discussion around the use of telemedicine for chronic condition management has also grown, and studies have shown similar outcomes for some conditions with telemedicine use, including diabetes and congestive heart failure. ${ }^{17}$ The increased utilisation has encouraged discussion around the integration of telemedicine into accreditation for healthcare providers, funding for telemedicine and redesigning clinical care models among other things. ${ }^{16}$

As a result of increased utilisation, new methods of triaging within telemedicine have emerged. For example, automated logic flows, also referred to as bots, have the ability to refer moderate and high-risk patients to triage lines that have nurses on them while also allowing video visits with providers virtually to avoid in-person interactions. $^{15}$

Barriers that currently exist to telemedicine that have been noted include a lack of education about the efficacy and safety of telemedicine in light of current circumstances, patient preferences regarding seeing their own provider as opposed to someone with whom they have no established relationship, a lack of understanding about how to access telemedicine visits, and a lack of knowledge about having telemedicine visits as an option. ${ }^{18}$ Barriers regarding a lack of clinician centric physical exam ${ }^{19}$ have been overcome in part in a few ways, including temperature taking via a home thermometer, noting a patient's general appearance via video, observations of a patient's respirations (including accessory respiratory muscle involvement, effort of breathing, and speech), presence of a patient cough (be it dry or productive), oropharynx observation via video and patient-directed lymph nodes to assess for notable lymphadenopathy. ${ }^{18}$

\section{CMS, Medicare and Medicaid regulations}

The CMS have recently expanded access to Medicare telemedicine. This expansion has allowed healthcare services to continue while preventing the spread of the virus. During the current public health emergency, all Medicare beneficiaries across the USA can receive Medicare telemedicine care at the same rate as in-person healthcare visits. Telemedicine may be offered to new or established patients by clinicians, and a broad range of clinicians may provide services through telemedicine. Finally, some telephone based medical services may be covered. ${ }^{20}$

Regarding Medicaid, states have the ability to determine the following regarding telemedicine: whether or not to use telemedicine, services that may be covered by Medicaid, where in a state it may be used, how telemedicine is implemented, what types of healthcare providers can deliver their services through telemedicine so long as they are both qualified and recognised according to Medicaid's federal and state statutes and regulations, and reimbursement rates for telemedicine visits. States are not required to submit amendments to pay for services should the services provided via telemedicine be the same as in an in-person setting. A state plan amendment would need to be submitted if services differed, however. Regarding ancillary costs that may arise as a result of telemedicine utilisation, such as technical support services and obtaining equipment for the instatement and delivery of telemedicine services, a state may pay for costs. ${ }^{20}$ It should be noted that copays have been waived for telemedicine visits by the CMS as well as some commercial healthcare plans in this time of need. ${ }^{18}$

\section{IT sector integration in healthcare}

Healthcare providers have been able to communicate with patients $24 / 7$ via webcam-enabled computers and smartphones. ${ }^{15}$ Telemedicine visits may include video communication but may also include text, email and mobile-phone applications as well as wearable devices and chatbots. ${ }^{3}$ Technical fees have been incorporated in Medicare and Medicaid coverage as a result of the IT sector integration into healthcare. ${ }^{20}$ Payment rules should allow for the creative implementation of current and rising digital technology, such as voice-interface services, mobile sensors, smartwatches and thermometers. $^{3}$ Moreover, there has been encouragement to consider digital services interstate commerce rather than intrastate to allow for federal jurisdiction over state jurisdiction regarding telemedicine; this may allow for a more cohesive set of rules to arise in this up-andcoming market. ${ }^{3}$ Improvements in technology have also improved current practice and created space for the future expansion of telemedicine. Namely, an increase in dependability, decrease in cost, improvement in audio and video quality, and emergence of products that reflect the clinical setting by incorporating virtual waiting rooms among other things have improved the ease and potential scalability of video telemedicine visits. ${ }^{21}$ 
Telemedicine has been shown to have lower transaction costs compared with in-person based care. ${ }^{21}$ The most cost-effective applications have been previously reported to be those that are paid for by insurers and have included radiology, psychiatry, home healthcare and prisoner healthcare in the past. ${ }^{22}$ Some services that can be used for telemedicine visits, such as Apple FaceTime, Google Hangouts, Facebook Messenger and Skype can be used at low or no cost, and recent liberalisations to HIPAA compliance guidelines for the COVID-19 pandemic by the US Department of Health and Human Services has made the aforementioned video systems means of telemedicine video visits. ${ }^{23}$ It has been suggested that technology companies who have the capabilities to create telemedicine devices that function as 'plug-and-play' devices at low costs to cover gaps that may exist in telemedicine. ${ }^{23}$ Telemedicine has been a cost effective and safe way to maintain healthcare through the COVID-19 pandemic.

One particular sector that has the greatest potential to benefit from increased utilisation of telehealth services is Rural America. According to the US Census Bureau, ${ }^{24}$ one in five Americans lives in rural areas. Although most rural Americans do have health insurance, $26 \%$ of these patients feel that they do not have appropriate access to healthcare. ${ }^{25}$ Reasons for this include both inability to afford care and difficulty accessing care due to distance or lack of a local provider who accepts the patient's insurance. ${ }^{25}$ Furthermore, nearly $8 \%$ of rural Americans report hospital closures in their local communities in the past few years. ${ }^{25}$ Further complicating matters for America's rural population is the looming physician shortage. According to the Association of American Medical Colleges(AAMC), ${ }^{26}$ the USA will have a shortage of nearly 122000 physicians by 2032 . While, this physician shortage will affect all Americans, rural and underserved healthcare regions will be hit the hardest. This may be due in part to difficulty recruiting and retaining physicians at rural hospitals. The current ratio of primary care physicians to patients in rural areas is 39.8 per 100000 compared with 53.3 per 100000 in urban areas. ${ }^{27}$ While the shortage must eventually be corrected, in the meantime it is vitally important to maximise the efficiency of the current workforce. Telemedicine can help do this by allowing physicians to see more patients by filling in time gaps in their day with virtual visits, providing rural hospitals with access to specialist care and even connecting with physicians in other countries through international telemedicine. Clearly, there is an urgent need for intervention that can improve access to quality, affordable care for these Americans.

Telemedicine has the potential to be a major part of the solution for rural patients. Currently, $85 \%$ of rural adults report using the internet, and $71 \%$ of rural adults own a smartphone. ${ }^{67}$ Telemedicine can act as an alternative option for these patients who already lack appropriate access to care. As discussed previously, many specialties are currently engaging in the use of telemedicine, and this can allow for the establishment of both primary and specialty care for those living in rural and underserved areas. Telemedicine has been shown to improve access to healthcare in these populations through a reduction in travel burden and decreased cost of care. ${ }^{28}$ In addition, the implementation of telemedicine in these areas can increase patient education and improve health outcomes via remote management of chronic conditions. There are, however, barriers to the implementation of telehealth services in rural America. These include limited insurance coverage for telehealth services under Medicare/Medicaid, issues with licensure including physicians inability to provide care across state lines, and a relative lack of technological advancements and RPM devices in rural vs urban America. ${ }^{10}$ An urge to develop solutions to these barriers in the post pandemic world can greatly improve the quality of healthcare offered in rural America.

The success of telemedicine as a means of providing high-quality healthcare has been well documented in recent studies. In the context of primary care, Powell et $a l^{29}$ interviewed patients following telemedicine visits with their primary care providers and found that all patients interviewed found telemedicine visits to be satisfactory for their primary care needs, and further, a majority stated that they would prefer to use telemedicine rather than in-person visits in the future. A study measuring the feasibility of using smartphones for surgical wound follow-up postappendectomy found that the sensitivity and specificity for detecting surgical wound complications was $100 \%$ and $91.67 \%$, respectively, using email imaging and patient questionnaires. ${ }^{30}$ Furthermore, a study using text messaging as a means of follow-up after colorectal surgery found that throughout the study, no postoperative complication was unrecognised, and even suggested that follow-up questionnaires regarding symptomatology via text messaging could potentially replace the traditional postoperative follow-up visit. ${ }^{31}$ Lastly, applications of telemedicine such as Tele-stroke services to provide stroke expertise to hospitals lacking experts in stroke care have been extremely successful and continue to grow in the USA. ${ }^{32}$

A number of specialties have taken to telemedicine to provide care for their patients; however, some use telemedicine more than others. Cardiologists, radiologists and psychiatrists are among those specialists who use telemedicine services most frequently. ${ }^{11}$ In the midst of the current pandemic, all medical specialties are being forced to adapt and change in order to continue to provide care to patients while maintaining social distancing. This increase in the use of telemedicine services may act as a turning point for the utilisation of telemedicine services in the USA moving forward. As organisations such as the American Medical Association and American Telemedicine Association continue to advocate for the use of telemedicine during the current pandemic, telemedicine may finally come to the forefront of healthcare heading into the future. American Telemedicine Association ${ }^{33}$ said of the current pandemic: 'Something like having to 
stay home could springboard telehealth tremendously, because when we get over this-and we will-people will have had that experience and they'll be saying, 'Well, why can't I do other aspects of my healthcare that way?'. Based on the data from prior studies, it is safe to say that telemedicine has already proven to be equal to in person care in certain aspects of various specialties, and we can only assume that with the increased utilisation of these services we will continue to see improvements moving forward.

From the patient's standpoint, the primary aim of telemedicine is to increase access to care and enhance the convenience of healthcare delivery. Virtual appointments may provide specialty care to populations where it otherwise may not be available, such as those living in rural areas, deployed on military assignments or in prisons. Furthermore, it can bring care to patients who may have difficulty making it to their appointments, such as the elderly, disabled or those lacking sufficient transportation.

Telemedicine can also greatly decrease the time it takes to receive medical care. Between travel time to healthcare facilities, waiting room time, and time actually obtaining medical care, Americans spend an average of 123 min per visit, with an average face-to-face time with a physician of 20.5 min. ${ }^{34}$ Telemedicine appointments may virtually eliminate travel and waiting times, dramatically increasing the proportion of patients' face-to-face time with their physicians.

In addition, telemedicine may play a role in reducing healthcare costs to the patient. Appropriate remote monitoring of patients' well-being and chronic medical conditions can help patients avoid costly emergency department visits or hospitalisations. Telemedicine has been found to save patients an average of US $\$ 19-$ US $\$ 121$ per visit, with savings primarily generated by avoidance of emergency department visits. ${ }^{35}$

Telemedicine also offers a number of advantages from the healthcare provider's perspective. Through monitoring patients in their home environments, physicians may be provided with deeper insights into patients' social determinants of health. This may be through exposure to the patients' family dynamics, economic barriers, and safety of living environments.

In addition, telemedicine may enhance provider productivity and provide new revenue streams. Similarly to the way telemedicine decreases a patient's need to travel, virtual visits may benefit providers who spend time travelling between several healthcare facilities to provide care. Likewise, new revenue streams may be created by expanding a practice's reach into new communities without the need to move locations. In addition, the option of telemedicine services within a practice may attract new consumers who would otherwise be unwilling to seek medical care.

Telemedicine may further ease strain on the healthcare system by managing capacity and cutting down on healthcare costs. One study estimates that by reducing $1 \%$ of emergency department visits through the use of telemedicine, we could see annual savings of US $\$ 101920000 .^{9}$ It is especially important to reduce emergency department and urgent care visits during the COVID-19 pandemic, not only for the patient's protection, but to ease strain on an already overburdened healthcare system.

There are other avenues in which telemedicine can reduce healthcare spending. For example, the Bureau of Justice Statistics reported that in 2011, US $\$ 7.7$ billion was spent on inmate healthcare. The Texas Department of Criminal Justice ${ }^{36}$ has shown that telemedicine can dramatically reduce these costs by stopping $85 \%$ of inmates from needing to leave the prison for healthcare and ultimately saving US\$780 million over 14 years.

It is also important to mention the impact telemedicine may have on healthcare's carbon footprint. Replacing in-person visits with telemedicine appointments has the potential to decrease carbon emissions via reducing travel to and from appointments. This may serve to argue for the use of telemedicine not only for those who lack access to traditional medical care, but as a staple for ordinary healthcare delivery.

A major disadvantage of telemedicine, which is to be expected with any evolving technology, is lack of consumer awareness regarding their access to it, its services and its cost. According to a 2019 J.D. Power Telehealth Satisfaction Study, ${ }^{9} 74.3 \%$ of consumers are unaware or believe their health system does not offer telemedicine services. Most notably, in rural and suburban areas $72 \%$ and $70.3 \%$ of consumers, respectively, were unaware of the services. ${ }^{9}$ Additionally, $0 \%$ of customers who indicated their health as 'poor' reported using telemedicine in the past year. ${ }^{9}$ Thus, it appears the patients who may benefit the most from its services are using it the least. Additionally, consumers' perspectives on the cost of telemedicine varies, with $13.3 \%$ believing telemedicine to be more expensive than a doctor's office visit and $48 \%$ believing it to be less expensive. ${ }^{9}$

Furthermore, many of those who lack access to traditional healthcare may lack access to telemedicine capabilities as well. Those who are older, live in rural areas, have less education, and have more chronic conditions are less likely to have access to the internet than their counterparts. ${ }^{10}$ A systematic review uncovered the most frequently reported barriers to telemedicine use from both patient and provider perspectives. The most common barriers from the patient's perspective involved age, level of education, computer literacy, bandwidth and unawareness of services whereas providers struggled with cost, reimbursement, legal liability, privacy confidentiality, security of data, effectiveness, old equipment and efficiency. ${ }^{37}$

A number of social implications may arise with increasing use of telemedicine. The rapid progression of the digital age has already led to patient complaints about physicians spending more time looking at computer screens than their patients. As telemedicine replaces more in-person visits, the patient-physician interaction could be further jeopardised. Since not all health systems offer telemedicine services at this time, a patient's utilisation 
of a telemedicine service could mean having to see a new provider, creating discontinuity of care.

Another concern when considering telemedicine and mobile health is the security of personal health information. Patients and providers may use websites or applications to share health data including diagnoses, results and in the provision of care. ${ }^{38}$ Sunyaev $e t a l^{39}$ investigated the privacy policies of 600 of the most commonly used mobile health apps, finding that only $30.5 \%$ of these had privacy policies. In addition, they found that of those who did have privacy policies, the majority required collegelevel literacy and $66.1 \%$ did not specifically address the app itself. ${ }^{39}$ This is a major problem when considering the widespread implementation of online healthcare services, because if patients are not able to access or understand privacy policies they will likely be reluctant to engage in the use of mobile health services. Moreover, issues with the security of personal health information being shared online makes telemedicine susceptible to cybercriminal activity. In order to provide telemedicine consultations, providers often use a variety of applications, devices and software programmes to connect with the patient. ${ }^{40}$ Issues that can make health information susceptible include lags in security updates, insecure connections and the use of public networks. In order to protect the integrity of patient data, a secure infrastructure allowing remote communication without reduction of security must be implemented. ${ }^{40}$

Finally, the lack of physical contact between patient and provider creates challenges when performing a remote physical examination. This is an implicit limitation of telemedicine that will affect certain medical specialties more than others. Cardiopulmonary examinations that rely heavily on auscultation and abdominal examinations that require palpation will be met with great difficulty, whereas specialties that rely on visual physical examination, such as dermatology or verbal communication, such as psychiatry, may be relatively spared from this limitation. However, new technology such as electronic stethoscopes and smartphone applications that measure patients' selfpalpation are addressing these more intimate aspects of the physical examination. ${ }^{41}$

With technology rapidly advancing, physician shortages increasing, our population ageing and the burden of the current pandemic on our healthcare system, it seems that utilisation of telemedicine may be more important than ever. The benefits of telemedicine are obvious when it comes to social distancing and decreased use of emergency departments, but barriers to telemedicine services including problems with availability and coverage of services contribute to the lack of widespread implementation. In order for telemedicine to continue to expand in the postpandemic world, steps towards increasing access and providing consumer education to be made.

As technology continues to advance, access to telemedicine services will likely become more widespread. As of 2019, 90\% of Americans are using the internet, a $38 \%$ increase since the year $2000 .{ }^{6}$ In addition, further advances in telemedicine will be made possible through the increasing use of smartphones. Today, $81 \%$ of Americans own smartphones, compared with just $35 \%$ in $2011 .^{7}$

Telemedicine's range will continue to expand, connecting patients and providers internationally as providers look to expand globally. This will not only help improve the physician shortage and mitigate the maldistribution of physicians in the USA, but may also provide patients with rare diseases alternative avenues to seek highly specialised care.

Implementation of telemedicine into management of chronic disease may prove vitally important for our healthcare system going forward. The $\mathrm{CDC}^{42}$ reports "chronic diseases such as heart disease, cancer and diabetes are the leading causes of death and disability in the USA (and) are also leading drivers of the nation's US\$3.5 trillion in annual healthcare costs.' Traditionally the management of chronic disease has relied on a series of return clinic visits scheduled at arbitrary times. Telemedicine now allows for shorter, more frequent virtual visits, with the ability of connecting multiple providers in the care of a patient. By frequent weight checks in a patient with congestive heart failure or regular blood glucose checks in a patient with diabetes, readmissions to the hospital and emergency department visits may be avoided, easing strain on the healthcare system and lowering the overall cost of chronic disease management.

\section{Limitations}

There are limitations to the current review. First, the review was performed as a narrative review rather than a systematic review of the literature. By nature, a systematic review has more rigorous and detailed methods for searching the literature, and therefore, some studies may have been missed. Additionally, our article does not discuss the current landscape of telemedicine-based training for primary care physicians.

\section{CONCLUSION}

Over the past several decades, we have been hit with numerous pandemics, including H1N1, Ebola, SARS-CoV, MERS-CoV, and currently, COVID-19. In the future, it is probable that more novel pandemics will arise. Prior to the current pandemic, issues with regulation and reimbursement have prevented telemedicine from being fully immersed into the healthcare landscape in the USA. The development and utilisation of telemedicine services is important, as these services allow us to continue to provide high-quality healthcare while maintaining the practice of physical distancing to prevent the spread of these viruses. The benefits of telemedicine include convenience, increased access to care from a distance, especially for patients living in rural areas, and decreased healthcare costs. Studies have shown that telemedicine appointments can be equal to in-patient visits in a variety of specialties. Continued research should be done to improve aspects of the physical examination for 
telemedicine visits, especially for specialties in which intimate patient contact is an important aspect of the physical exam. Now is the time for us to implement these services and make the usage of telemedicine mainstream. If we do this, we will be prepared for the next pandemic and the future of healthcare.

Contributors AK is credited with substantial contribution to the design of the work, literature review of all the sections discussed, the revision of critically important intellectual content, final approval of the published version, and agreement of accountability for all aspects of the work. MA, KD and FW are credited with substantial acquisition of the literature reviewed for the manuscript, drafting the manuscript, final approval of the version to be published, and agreement of accountability for all aspects of the work. ZE-A, JS and MA are credited with significant contribution to the acquisition of the literature reviewed, the revision of critically important intellectual content, final approval of the version to be published, and agreement of accountability for all aspects of the work. RCC and AKK are credited with significant contribution to the revision of the manuscript, mainly literature review and drafting of telemedicine in current scenario of COVID-19, telemedicine and the need of healthcare in rural America, and studies of outcomes of telemedicine, final approval of the version to be published, and agreement of accountability for all aspects of the work. SS and SC are credited with design of the work, drafting and revision of the manuscript, mainly advantages and disadvantages of telemedicine and future of telemedicine, final approval of the version to be published and agreement of accountability for all aspects of the work.

Funding The authors have not declared a specific grant for this research from any funding agency in the public, commercial or not-for-profit sectors.

Competing interests None declared.

Patient consent for publication Not required.

Ethics approval Our institution does not require ethical approval for literature reviews or review articles.

Provenance and peer review Not commissioned; externally peer reviewed.

Open access This is an open access article distributed in accordance with the Creative Commons Attribution Non Commercial (CC BY-NC 4.0) license, which permits others to distribute, remix, adapt, build upon this work non-commercially, and license their derivative works on different terms, provided the original work is properly cited, appropriate credit is given, any changes made indicated, and the use is non-commercial. See: http://creativecommons.org/licenses/by-nc/4.0/.

\section{ORCID iDs}

Michael Albosta http://orcid.org/0000-0003-4187-4911

Savneek Chugh http://orcid.org/0000-0002-0085-8272

\section{REFERENCES}

1 Telemedicine: centers for Medicare and Medicaid services;. Available: https://www.medicaid.gov/medicaid/benefits/telemedicine/ index.html

2 Waller M, Stotler C. Telemedicine: a primer. Curr Allergy Asthma Rep 2018;18:54.

3 Keesara S, Jonas A, Schulman K. Covid-19 and health care's digital revolution. N Engl J Med 2020;382:e82.

4 Hurst EJ. Evolutions in telemedicine: from smoke signals to mobile health solutions. J Hosp Librariansh 2016;16:174-85.

5 Zundel KM. Telemedicine: history, applications, and impact on librarianship. Bull Med Libr Assoc 1996;84:71-9.

6 Internet/Broadband fact sheet: Pew research center and American life project, 2019. Available: https://www.pewresearch.org/internet/ fact-sheet/internet-broadband/

7 Mobile fact sheet: Pew research center and American life project, 2019. Available: https://www.pewresearch.org/internet/fact-sheet/ mobile/

8 American Hospital Association. Fact sheet: telehealth, 2019. Available: https://www.aha.org/system/files/2019-02/fact-sheettelehealth-2-4-19.pdf

9 Telehealth satisfaction study: J.D. power, 2019. Available: https:// www.americantelemed.org/resources/telehealth-adoption-andusage/

10 Dorsey ER, Topol EJ. State of telehealth. N Engl J Med 2016;375:154-61.
11 Robeznieks A. Which Medical Specialties Use Telemedicine the Most? : American Medical Association, 2019. Available: https:// www.ama-assn.org/practice-management/digital/which-medicalspecialties-use-telemedicine-most

12 Toolkit on the right to health: United nations human rights office of the high commissioner. Available: https://www.ohchr.org/EN/Issues/ ESCR/Pages/Health.aspx

13 Public Health Institute Center for Connected Health Policy. State Telehealth Laws \& Reimbursement Policies, 2020. Available: https:// www.cchpca.org/sites/default/files/2020-05/CCHP_\%2050_STATE_ REPORT_SPRING_2020_FINAL.pdf

14 Oh J-Y, Park Y-T, Jo EC, et al. Current status and progress of telemedicine in Korea and other countries. Healthc Inform Res 2015;21:239-43.

15 Hollander JE, Carr BG. Virtually perfect? telemedicine for Covid-19. N Engl J Med 2020;382:1679-81.

16 Smith AC, Thomas E, Snoswell CL, et al. Telehealth for global emergencies: implications for coronavirus disease 2019 (COVID-19). J Telemed Telecare 2020;1357633X:20916567.

17 Flodgren G, Rachas A, Farmer AJ, et al. Interactive telemedicine: effects on professional practice and health care outcomes. Cochrane Database Syst Rev 2015;9:CD002098.

18 Portnoy J, Waller M, Elliott T. Telemedicine in the era of COVID-19. J Allergy Clin Immunol Pract 2020;8:1489-91.

19 Thirthalli J, Manjunatha N, Math SB. Unmask the mind! Importance of video consultations in psychiatry during COVID-19 pandemic [published online ahead of print, 2020 Jun 15]. Schizophr Res 2020;S0920-9964:30357-1.

20 Services CfMaM. State Medicaid and CHIP Telehealth Toolkit - Policy Considerations for States Expanding Use of Telehealth COVID-19 Version, 2019. Available: https://www.medicaid.gov/medicaid/ benefits/downloads/medicaid-chip-telehealth-toolkit.pdf

21 Greenhalgh T, Wherton J, Shaw S, et al. Video consultations for covid-19. BMJ 2020;368:m998.

22 Charles BL. Telemedicine can lower costs and improve access. Healthc Financ Manage 2000;54:66-9.

23 Calton B, Abedini N, Fratkin M. Telemedicine in the time of coronavirus. J Pain Symptom Manage 2020.

24 United States Census Bureau. 2010 census urban and rural classification and urban area criteria, 2019. Available: https://www. census.gov/programs-surveys/geography/guidance/geo-areas/ urban-rural/2010-urban-rural.htm

25 Life in rural America Part II: NPr, the Robert wood Johnson Foundation, and the Harvard T.H. Chan school of public health, 2019. Available: https://media.npr.org/documents/2019/may/NPR-RWJFHARVARD_Rural_Poll_Part_2.pdf

26 AAMC. New findings confirm predictions on physician shortage, 2019. Available: https://www.aamc.org/news-insights/press-releases/ new-findings-confirm-predictions-physician-shortage

27 Hing E, Hsiao C-J. State variability in supply of office-based primary care providers: United States, 2012. NCHS Data Brief 2014;151:1-8.

28 Kruse CS, Bouffard S, Dougherty M, et al. Telemedicine use in rural native American communities in the era of the ACA: a systematic literature review. J Med Syst 2016;40:145.

29 Powell RE, Henstenburg JM, Cooper G, et al. Patient perceptions of telehealth primary care video visits. Ann Fam Med 2017;15:225-9.

30 Segura-Sampedro JJ, Rivero-Belenchón I, Pino-Díaz V, et al. Feasibility and safety of surgical wound remote follow-up by smart phone in appendectomy: a pilot study. Ann Med Surg 2017;21:58-62.

31 Carrier G, Cotte E, Beyer-Berjot L, et al. Post-Discharge follow-up using text messaging within an enhanced recovery program after colorectal surgery. J Visc Surg 2016;153:249-52.

32 Wechsler LR, Demaerschalk BM, Schwamm LH, et al. Telemedicine quality and outcomes in stroke: a scientific statement for healthcare professionals from the American heart Association/American stroke association. Stroke 2017;48:e3-25.

33 Ducharme J. The coronavirus outbreak could finally make telemedicine mainstream in the U.S. TIME: Time Magazine, 2020.

34 Ray KN, Chari AV, Engberg J, et al. Disparities in time spent seeking medical care in the United States. JAMA Intern Med 2015;175:1983-6.

35 Nord G, Rising KL, Band RA, et al. On-Demand synchronous audio video telemedicine visits are cost effective. Am J Emerg Med 2019;37:890-4.

36 Smith D, Sorenson G, Lewis B. Why telemedicine, why now? GlobalMed, 2019. Available: https://www.americantelemed.org/ resources/why-telemedicine-why-now/

37 Scott Kruse C, Karem P, Shifflett K, et al. Evaluating barriers to adopting telemedicine worldwide: a systematic review. J Telemed Telecare 2018;24:4-12. 
38 Petersen C, DeMuro P. Legal and regulatory considerations associated with use of patient-generated health data from social media and mobile health (mHealth) devices. Appl Clin Inform 2015:6:16-26.

39 Sunyaev A, Dehling T, Taylor PL, et al. Availability and quality of mobile health APP privacy policies. J Am Med Inform Assoc 2015;22:e28-33.
40 Chilson M. Prognocis EHR, 2019. Available: https://prognocis.com/ telemedicine-and-cybersecurity-keeping-health-data-safe/

41 Ansary AM, Martinez JN, Scott JD. The virtual physical exam in the 21st century. J Telemed Telecare 2019;1357633X:19878330.

42 Centers for Disease Control and Prevention. About chronic diseases, 2019. Available: https://www.cdc.gov/chronicdisease/about/index. htm 